\title{
The challenge of achieving continuous quality improvement in Canadian pediatric care
}

\author{
Mary E. Brindle MD MPH
}

Cite as: CMAJ 2020 January 27;192:E79-80. doi: 10.1503/cmaj.200008

See related article at www.cmaj.ca/lookup/doi/10.1503/cmaj.190940

W ithin quality improvement, change may be easy, but large-scale change is hard, and sustaining such change is harder still. Enthusiasm for qualityimprovement initiatives frequently wanes, often because positive results are thought to be attributable to changes in global practice rather than successful implementation of initiatives. The findings of a linked longitudinal population-based cohort study that describes improved outcomes for premature infants through multimodal, continuous quality improvement clearly illustrates a success story in Canadian pediatric quality improvement. ${ }^{1}$ Lee and colleagues' platform of Evidence-based Practice for Improving Quality (EPIQ; www.epiq.ca) demonstrates a rare sustained achievement. Over several epochs of this national program, survival without major morbidity has consistently increased among premature infants, which illustrates the importance of commitment to ongoing improvement in national quality-improvement programs. ${ }^{1}$

Pediatric quality improvement has taken root in specific areas in Canada, with national strategies having developed in a few key disciplines, building on established databases and focusing on measurable, comparable and improvable outcomes. Multimodal and multidisciplinary strategies focusing on low-stakes, easily achievable care practices form the core of sustainable quality improvement. The Canadian Neonatal Network (www.canadian neonatalnetwork.org) was established in 1995 with a goal to improve Canadian neonatal outcomes. The network developed as a community of neonatal researchers and hosts a database that captures information on premature neonates receiving care across Canada. Premature infants are an optimal target for quality improvement, as they have high rates of adverse outcomes and are cared for in centralized units. The database of the Canadian Neonatal Network captures data on $80 \%$ of Canadian premature infants. Similar neonatal national databases exist worldwide, allowing for benchmarking of population-based outcomes for comparable health systems. ${ }^{2}$

Pediatric surgery is also developing national strategies for quality improvement, with somewhat less success. Some qualityimprovement initiatives have been adopted from US organizations such as the National Surgical Quality Improvement Program

\section{KEY POINTS}

- Effective quality improvement requires that short-term innovation be integrated swiftly into a strategy for long-term evolution.

- Multimodal and multidisciplinary strategies focusing on lowstakes, easily achievable care practices form the core of sustainable quality improvement.

- Large clinical databases, while helpful to the development and evaluation of national quality-improvement programs, do not of themselves ensure excellent outcomes.

- Failure is virtually ensured without a commitment to continuous improvement.

- A virtuous cycle can be achieved through commitment to continuous improvement that builds on positive results to reinforce the importance of quality improvement and gradually changes the culture of health care.

(NSQIP). Others have developed within Canada, such as the Canadian Pediatric Surgery Network (CAPSNet). Both NSQIP and CAPSNet provide platforms for quality-improvement initiatives. However, the existence of quality-improvement databases does not necessarily ensure excellent outcomes. The spread of NSQIP across Canadian pediatric surgical centres has been expensive and slow. Despite substantial investment in multidisciplinary practice change at the first Canadian pediatric NSQIP implementation site, assessment of its full impact on quality improvement is limited. ${ }^{3}$ The Canadian Pediatric Surgery Network has achieved greater national spread within limited areas of pediatric surgery, specifically, congenital diaphragmatic hernia and gastroschisis. Although CAPSNet provides a strong foundation for the development of practice guidelines, it has not yet driven quality improvement on a national level. ${ }^{4}$ Enhanced Recovery After Surgery is a high-value quality-improvement initiative within adult surgery that is only just starting to make inroads in pediatric care. ${ }^{5}$ In contrast, the Canadian Neonatal Network began its work in quality improvement early and has succeeded in improving outcomes because of a strategic initiative (EPIQ) that iteratively scaled up quality-improvement interventions over a period of 15 years. 
When attempting to sustain change on a large scale, health systems frequently, and almost inevitably, run into failure. A prominent example is the failure of the Surgical Safety Checklist in Ontario. Use of this checklist was associated with improved outcomes in many international studies ${ }^{6}$ but had little impact when introduced in Ontario, especially for children. ${ }^{7}$ The failure of the checklist in Ontario can be contrasted with the success of EPIQ in several ways but particularly as it relates to the importance of a centralized and longitudinal implementation plan. Use of the surgery checklist was mandated in Ontario with variable local strategies for implementation and no centralized approach or feedback on effectiveness. ${ }^{8}$ The EPIQ program, however, was introduced strategically. Built on a foundation of reliable data from the Canadian Neonatal Network, EPIQ started small, as a cluster randomized controlled trial (RCT) comparing 6 neonatal intensive care units (NICUs) that adopted strategies to improve bronchopulmonary disease with 6 NICUs that adopted strategies to reduce nosocomial pneumonia. With evidence of success (particularly at reducing bronchopulmonary disease), EPIQ expanded, with a goal for scale, spread and sustainability. The elements contributing to success included reliable data, multidisciplinary teams, a centralized strategy for implementation (albeit with variations of implementation by location) and a commitment to continuous improvement.

From 2004 to 2017, EPIQ moved through a series of epochs. After the initial RCT, the program's strategies spread across Canada's NICUs with a focus on dissemination and evaluation. The program expanded its scope, targeting key morbidities through specific evidence-based practices. In 2011, data collection was expanded to include neurodevelopmental feedback. In 2015, EPIQ encouraged the spread of bundles of care, sentinel event reviews, site visits and new outcome targets. None of the interventions were particularly innovative or earth-shattering, but, before this program, these evidence-based practices were not reliably performed, and outcomes were not immediately apparent. ${ }^{9}$ The program's approach continues to show measurable successes, with clear clinical benefits encouraging the adoption of new quality-improvement initiatives in a virtuous cycle of quality-improvement culture supporting qualityimprovement initiatives.

There are challenges, in moving from an RCT to a longitudinal cohort study, in showing which improvements arise from a system of continuous quality improvement and which have resulted from a gradual shift in culture or dissemination of knowledge. National quality-improvement programs like EPIQ must avoid "taking credit for the weather." Quality-improvement programs can be benchmarked against systems not engaged in enhanced quality improvement - as in the comparison of the Vermont Oxford Network's success in reducing neonatal morbidity over 10 years with smaller improvements seen in similar NICUs in
North America over the same era ${ }^{10}$ - but this requires similar systems with similar data. Randomized trials evaluating the impact of elements of quality improvement can provide evidence of their benefits, but trials are costly, time consuming and narrowly focused. Sustaining longitudinal quality improvement requires reliable data, evidence of value and investment in a culture that supports quality improvement.

The achievements of EPIQ challenge what we traditionally expect from the medical literature: a single intervention delivered over a short period that produces a beneficial outcome. The program's achievements are broad in scope, incremental and continuous; its ongoing evolution is the secret to its success. Lee and colleagues' study serves as a reminder that effective quality improvement requires that short-term innovation be integrated swiftly into a strategy for long-term evolution.

\section{References}

1. Lee SK, Beltempo M, McMillan DD, et al.; Evidence-based Practice for Improving Quality Investigators. Outcomes and care practices for preterm infants born at less than 33 weeks' gestation: a quality-improvement study. CMAJ 2020; 192:E81-91.

2. Helenius K, Sjörs G, Shah PS, et al.; International Network for Evaluating Outcomes (iNeo) of Neonates. Survival in very preterm infants: an international comparison of 10 national neonatal networks. Pediatrics 2017;140:pii: e20171264.

3. Skarsgard ED, Bedford J, Chan T, et al. ACS national surgical quality improvement program: targeting quality improvement in Canadian pediatric surgery. J Pediatr Surg 2014;49:682-7.

4. Butler AE, Puligandla PS, Skarsgard ED. The Canadian Pediatric Surgery Network (CAPSNet): lessons learned from a national registry devoted to the study of congenital diaphragmatic hernia and gastroschisis. Eur J Pediatr Surg 2015; 25:474-80.

5. Brindle ME, Heiss K, Scott MJ, et al.; Pediatric ERAS (Enhanced Recovery After Surgery) Society. Embracing change: the era for pediatric ERAS is here. Pediatr Surg Int 2019;35:631-4.

6. Bergs J, Hellings J, Cleemput I, et al. Systematic review and meta-analysis of the effect of the World Health Organization surgical safety checklist on postoperative complications. Br J Surg 2014;101:150-8.

7. O'Leary JD, Wijeysundera DN, Crawford MW. Effect of surgical safety checklists on pediatric surgical complications in Ontario. CMAJ 2016;188:E191-8.

8. Urbach DR, Govindarajan A, Saskin R, et al. Introduction of surgical safety checklists in Ontario, Canada. N Engl J Med 2014;370:1029-38.

9. Aziz K, McMillan DD, Andrews W, et al.; Canadian Neonatal Network. Variations in rates of nosocomial infection among Canadian neonatal intensive care units may be practice-related. BMC Pediatr 2005;5:22.

10. Horbar JD, Carpenter JH, Badger GJ, et al. Mortality and neonatal morbidity among infants 501 to 1500 grams from 2000 to 2009. Pediatrics 2012;129: 1019-26.

\section{Competing interests: None declared.}

This article was solicited and has not been peer reviewed.

Affiliation: Departments of Surgery and Community Health Sciences, Cumming School of Medicine, University of Calgary, Calgary, Alta.

Correspondence to: Mary Brindle, Mary.Brindle@ahs.ca 